\title{
Chronic myelomonocytic leukemia: the role of bone marrow biopsy immunohistology
}

\author{
Attilio Orazi ${ }^{1}$, Ronald Chiu ${ }^{1}$, Dennis P O’Malley ${ }^{1}$, Magdalena Czader ${ }^{1}$, Susan L Allen ${ }^{1}$, \\ Caroline $\mathrm{An}^{1}$ and Gail $\mathrm{H}$ Vance ${ }^{2}$ \\ ${ }^{1}$ Clarian Pathology Laboratory, Department of Pathology and Laboratory Medicine, Indiana University School \\ of Medicine, Indianapolis, IN, USA and ${ }^{2}$ Department of Medical and Molecular Genetics, Indiana University \\ School of Medicine, Indianapolis, IN, USA
}

\begin{abstract}
The World Health Organization criteria for diagnosing chronic myelomonocytic leukemia (CMML) are largely based on findings observed in the peripheral blood and bone marrow aspirate. A specific diagnostic role for the bone marrow biopsy has not been adequately explored. We examined whether bone marrow biopsy supplemented by immunohistochemistry may be helpful in distinguishing CMML from cases of chronic myelogenous leukemia and atypical chronic myeloid leukemia (aCML). We immunostained 25 cases of CMML with paraffin reactive antibodies which included CD68 (KP1), CD68R (PG-M1), and CD163, and compared the results with those observed in six cases of chronic myelogenous leukemia and in three cases of atypical CML. In addition, we examined whether CD34 immunohistochemistry could be useful in separating cases of CMML with less than $10 \%$ blasts (type-1) from cases of CMML with blasts accounting for 10-19\% (type-2), and cases of CMML in acute transformation to acute myeloid leukemia (blasts $\geq 20 \%$ ). The presence of nodules of plasmacytoid monocytes was investigated by CD123 staining. CD42b was used to highlight abnormal megakaryocytes. Our results demonstrate significant differences between the groups. CD34 analysis allowed separating CMML type-1 from type-2 and the former from CMML in acute transformation. CD123-positive plasmacytoid monocyte nodules were found only in CMML and not in the other two disease groups. Overlap between CMML and the other two groups were observed with CD68 immunostaining. CD68R was more restricted to bone marrow macrophages and monocytes than CD68, but the differences between CMML and chronic myelogenous leukemia or atypical CML were still not significant. Although CD42b immunostaining facilitated the detection of dwarf megakaryocytes often present in CMML, the distinction between those and the small forms seen in chronic myelogenous leukemia was still problematic.
\end{abstract}

Modern Pathology (2006) 19, 1536-1545. doi:10.1038/modpathol.3800707; published online 13 October 2006

Keywords: chronic myelomonocytic leukemia; acute transformation; chronic myelogenous leukemia; atypical chronic myeloid leukemia; immunohistochemistry; plasmacytoid monocyte

Chronic myelomonocytic leukemia (CMML) is a clonal hematopoietic malignancy which is characterized by hybrid features, of both a myeloproliferative and a myelodysplastic syndrome.$^{1-4}$ According to the World Health Organization (WHO) criteria, the diagnosis of CMML requires a persistent peripheral blood monocytosis of more than $1 \times 10^{9} / 1$, with a percentage of monocytes greater than $10 \%$ of the white blood cell count (WBC). The peripheral blood may demonstrate dysplastic changes typical of the myelodysplastic syndromes, or dysplastic

Correspondence: Dr A Orazi, MD, FRCPath (UK), Clarian Pathology Laboratory, Department of Pathology and Laboratory Medicine, Indiana University School of Medicine, 350 West 11th Street, Room 5034, Indianapolis, IN 46202-5200, USA.

E-mail: aorazi@iupui.edu

Received 6 February 2006; revised 25 August 2006; accepted 29 August 2006; published online 13 October 2006 changes may be minimal and limited to the monocytes; mild anemia is usually seen.

The bone marrow of CMML is usually hypercellular and in most cases, demonstrates a significant degree of granulocytic proliferation. ${ }^{1-3}$ Erythroid precursors and megakaryocytes may demonstrate prominent dysplastic changes, but these cell types are often normal in appearance.

Several studied have shown the importance of blast enumeration in CMML, their excess being associated with a poorer prognosis. ${ }^{3,5}$ On the basis of these studies, the WHO classification scheme subdivides CMML into two subcategories, depending on the number of blasts found in the peripheral blood and bone marrow. These include: CMML type- 1 with blasts $<5 \%$ in the blood or $<10 \%$ in the bone marrow, and CMML type-2 which is characterized by blasts $5-19 \%$ in the blood or $10-19 \%$ in the bone marrow, or when Auer rods are present and the 
blast count is less than $20 \%$ in blood or marrow. If blasts and promonocytes account for $\geq 20 \%$ of the bone marrow differential count, the diagnosis should be acute myeloid leukemia rather than CMML. ${ }^{1}$

Besides the assessment of the blast number and the presence and amount of dysplasia, it is unclear how bone marrow morphology can be useful in distinguishing CMML from other myeloid neoplasms, chronic myelogenous leukemia (CML) and atypical chronic myeloid leukemia (aCML) in particular. The two latter entities are also characterized by a degree of myeloid proliferation similar to that seen in most cases of CMML. ${ }^{1,8,9}$

Although the presence of monocytosis in peripheral blood is necessary for the diagnosis of CMML, a similar criterion has not been proposed for the bone marrow, in spite of the assumption than monocytic proliferation is present in the marrow as well. The rationale of this is that the presence of pronounced granulocytic proliferation makes it difficult to distinguish the monocytic population from the granulocytic precursors, myelocytes, and metamyelocytes in particular. ${ }^{1}$ To overcome this difficulty, the use of cytochemical stains for monocytes such as naphtyl butyrate esterase, or naphtyl acetate esterase has been advocated. ${ }^{1,6}$

The role of bone marrow biopsy in this condition is even less clear than that of the marrow aspirate. Besides the detection of an increased marrow cellularity owing to myeloid proliferation and the identification of fibrosis, additional diagnostic roles have not been explored. Bone marrow biopsy could be potentially more useful than the bone marrow aspirate in the presence of marrow fibrosis, which may cause a spurious dilution of the marrow cellularity owing to 'contamination' of the sample with peripheral blood. ${ }^{7}$ Potentially, immunohistochemistry applied to the bone marrow biopsy should provide a reliable tool for the 'in situ' identification of monocytes and monocytic cells, as well as facilitate the identification of plasmacytoid monocytes nodules, a relatively common finding in CMML. ${ }^{1}$

In this study, we examined whether bone marrow histology, supplemented by immunohistochemistry for monocytic markers may be helpful in distinguishing CMML from chronic myelogenous leukemia and atypical CML. We have also investigated CD34 immunostaining of bone marrow biopsy as a tool to separate CMML type- 1 from cases of CMML type-2 and CMML in acute transformation to acute myeloid leukemia (Figures 1-3).

\section{Materials and methods}

The materials studied included 25 cases of CMML, (20 cases of CMML type- 1 and five cases of chronic myelomonocytic leukemia type-2), six cases of chronic myelogenous leukemia in stable phase, and three cases of atypical CML. Additionally, a series of seven cases of CMML in acute transformation to acute myeloid leukemia were also included. For the purpose of this study, CMML in acute transformation to acute myeloid leukemia was defined by the presence of $\geq 20 \%$ marrow blasts in a patient with a previously documented diagnosis of CMML.

The cases were obtained from the files of the Department of Pathology, Indiana University School of Medicine/Clarian Health Partners, Indianapolis, Indiana. All cases were consecutively diagnosed within a period of approximately 10 years. Only 'de novo' (ie non-therapy-related) myeloid neoplasms were included. Peripheral blood smear, bone marrow aspirate smear, and paraffin-embedded bone marrow biopsy and/or particle sections were available for all cases used in this study. The diagnostic categorization of these cases was based on a comprehensive multiparametric evaluation, which included a combination of clinical characteristics, morphology, immunohistochemistry, and when available, flow cytometry and cytogenetics, in accordance with the diagnostic guidelines for CMML, chronic myelogenous leukemia, and atypical CML outlined in the current WHO classification. ${ }^{1}$

\section{Peripheral Blood and Bone Marrow Aspirate Evaluation}

A Wright-stained peripheral blood smear was evaluated and the percentage of circulating blasts as well as the absolute value and relative proportion of monocytes determined. Bone marrow aspirate smears were examined and a 300 cells differential count obtained. In 17 cases, naphtyl butyrate esterase was performed and used to assess the frequency of bone marrow monocytes.

\section{Histologic Evaluation}

In each case, hematoxylin-eosin-stained sections of the bone marrow biopsy and/or clot preparation was examined. In histology sections, blasts were defined as cells with high nuclear:cytoplasmic ratio, dispersed nuclear chromatin, and scant cytoplasm. A comparison 'side by side' with a corresponding section stained with CD34 (see below) was particularly helpful in confirming the increased marrow blast percentage seen in the aggressive cases of CMML (ie CMML type-2 and CMML in acute transformation). In addition, in each case, a section of the bone marrow biopsy was stained with Gomori and the degree of reticulin fibrosis was measured, according to the system originally proposed by Manoharan et $a l^{10}$ on a scale of $0-3+$. It should be noted that $4+$ fibrosis (presence of mature collagen) can be established only by the application of a trichrome stain, which was not performed for the purpose of this study. 

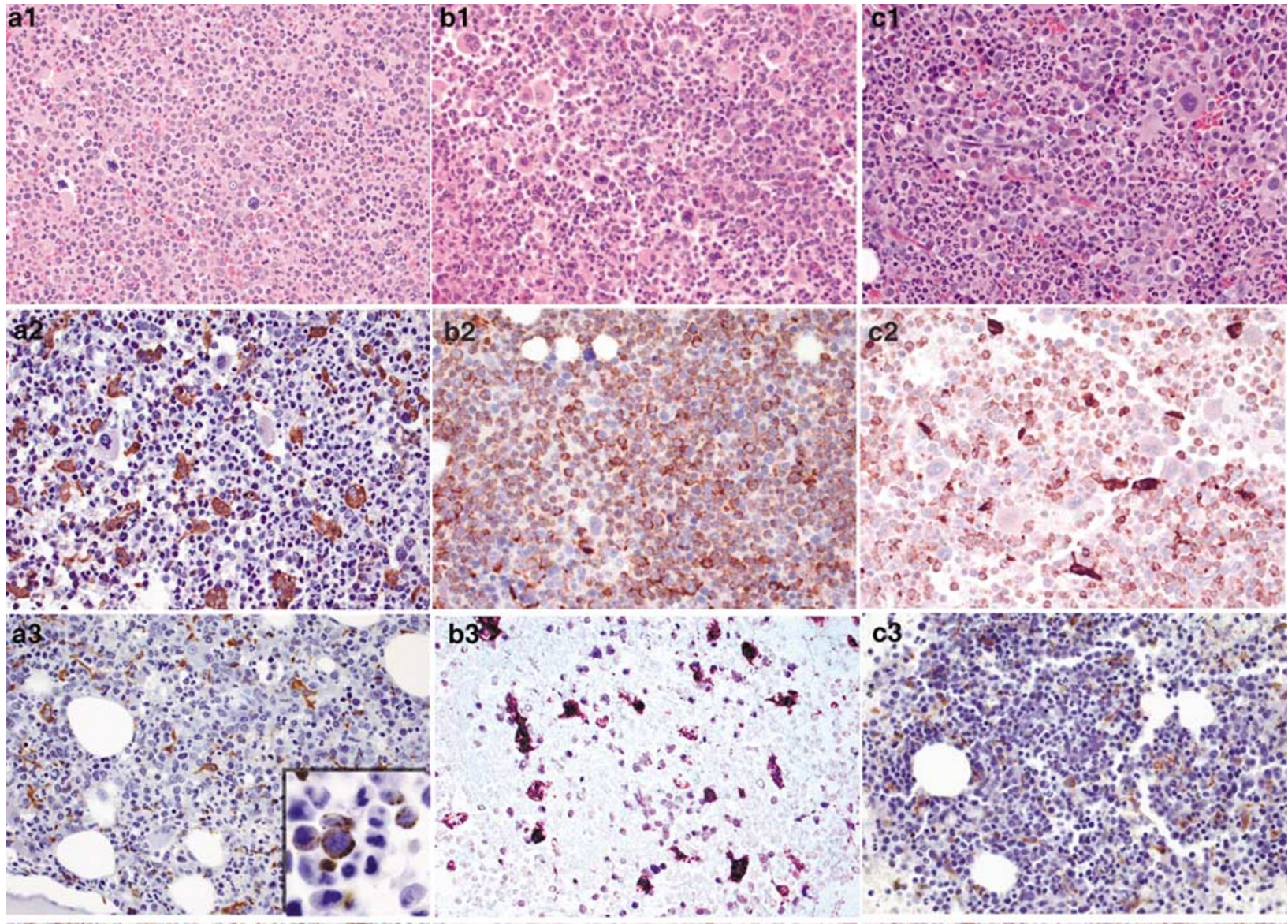
*is ing

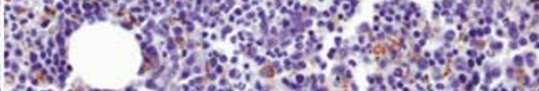
a

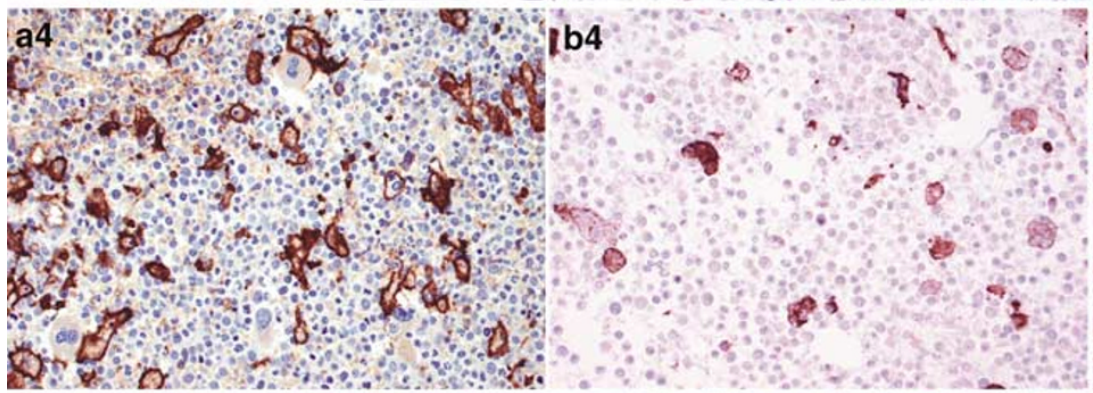

a5
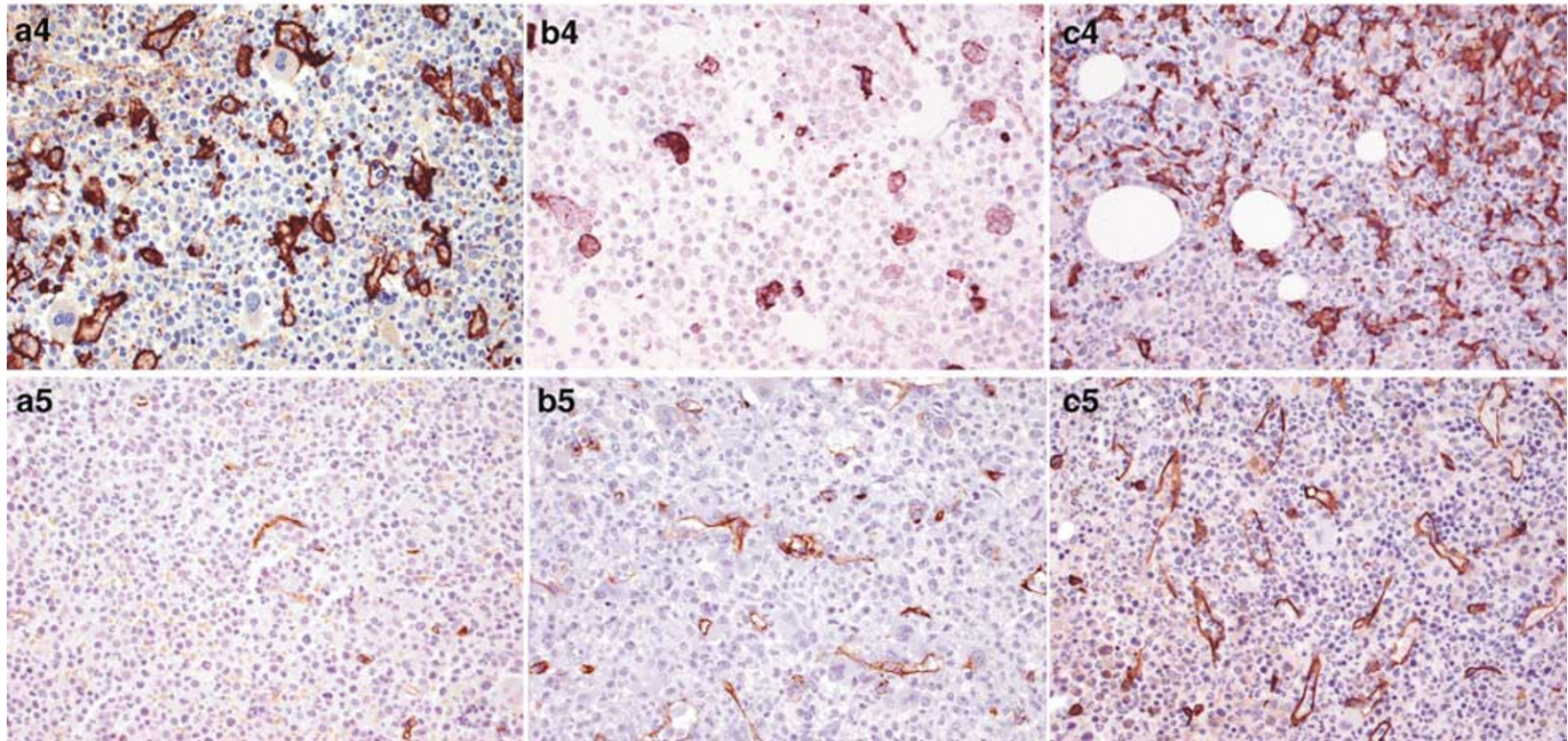

Figure 1 a1-5, CMML. b1-5, chronic myelogenous leukemia (CML). c1-5, atypical chronic myeloid leukemia (aCML). a1-H\&E of bone marrow section in CMML-1. Note the dwarf size megakaryocytes. b1-H\&E section of CML. Small, unilobate megakaryocytes are typically seen in CML. c1-H\&E section of aCML. Although there are subtle morphologic differences, the core biopsy findings of these three disorders (eg CMML, CML, and aCML) are quite similar. a2-CD68 (KP1) staining in CMML. Scattered myeloid cells and marrow macrophages are positively stained. b2-CD68 (KP1) staining in CML. Strong diffuse staining is seen in myeloid elements. c2-CD68 (KP1) staining in aCML. Diffuse staining is also seen. a3-CD68R (PGM-1) staining in CMML. CD68R-positive bone marrow macrophages are seen. Inset: note the few positive monocytes also stained. b3-CML and c3-aCML, CD68 (PGM-1) staining. Only marrow macrophages stain positively. a4-CMML, b4-CML, c4-aCML, CD163 stained mostly bone marrow macrophages in all three groups. a5-CMML, no increase in blasts are identified by CD34 staining in this case of CMML. b5-CML and c5-aCML, rare blasts stain for CD34. Note the increased vascular density highlighted by CD34. 


\section{Immunoperoxidase Methods}

Formalin-fixed, nitric acid decalcified, paraffinembedded bone marrow biopsies and/or particle sections were used for immunoperoxidase staining. This was performed by an automated immunostainer (DAKO, Carpinteria, CA, USA) using a standard streptavidin-biotin-peroxidase complex technique. The progenitor/early precursor associated antibody CD34 (QBEND10), an antigen expressed on blasts in most cases of myelodysplastic syndromes and myelodysplastic/myeloproliferative diseases ${ }^{7,11}$ and antibodies reactive with histiocytes/monocytes

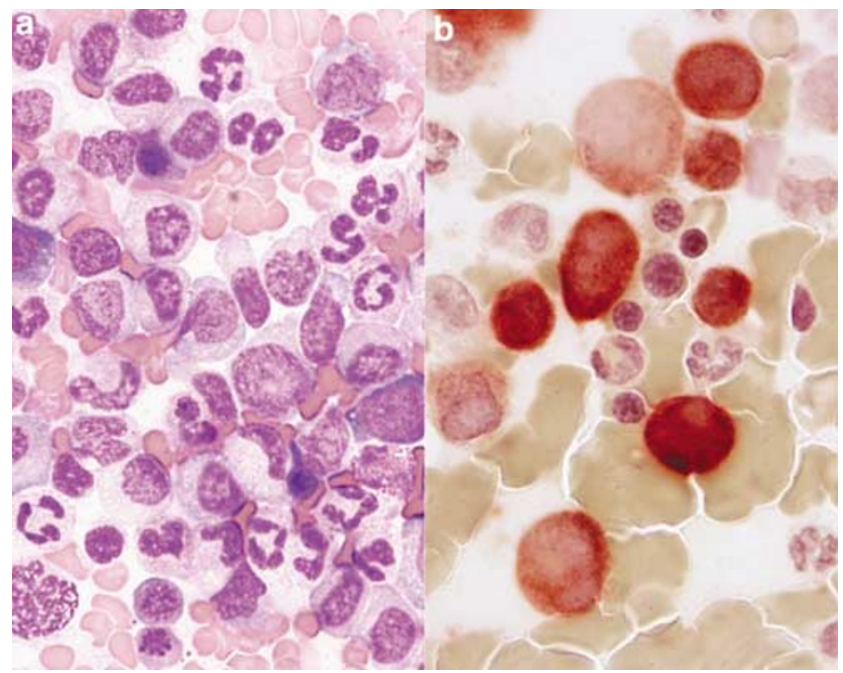

Figure 2 (a) Bone marrow aspirate smear of CMML. Numerous neutrophils and maturing myeloid elements are identified. (b) Cytochemical staining for naphtyl butyrate esterase highlighting monocytic elements in the aspirate smear. were investigated. The latter group included the following markers: CD68 (KP1), CD68R (PG-M1), and CD163 (10B6). ${ }^{12-15}$ The presence of nodules of plasmacytoid monocytes was investigated by CD123 (IL-3R-alpha) immunostaining. This antibody is considered as the most reliable marker to identify plasmacytoid monocytes present in myeloid neoplasms. ${ }^{16,17}$ CD42b (MM2/174), a marker expressed on megakaryocytes, was used to highlight the presence of dysplastic megakaryocytic forms, an approach that was recently found useful in a study from our group. ${ }^{7}$ Briefly, endogenous peroxidase activity was blocked with $3 \%$ hydrogen peroxide in methanol, and endogenous biotin activity was blocked using avidin and biotin. The peroxidase activity was developed with 3,3-diaminobenzidene, and counterstained with hematoxylin. Positive and negative controls were prepared and they stained appropriately. The immunohistochemical results were expressed as mean percentage, plus/minus standard deviation, ( \pm s.d.), of marrow cells reactive with the given antibody. Student's $t$-test was used to compare the results observed in the various groups.

\section{Cytogenetic Methods}

For bone marrow aspirate specimens, the cell culture, harvesting, and GTG-banding karyotype methods were performed as described previously. ${ }^{18}$

\section{Results}

\section{Patient Population}

The 32 patients in the CMML group ranged in age from 39 to 89 , their median age was 68 years; 19 were males and 13 were female. The six chronic
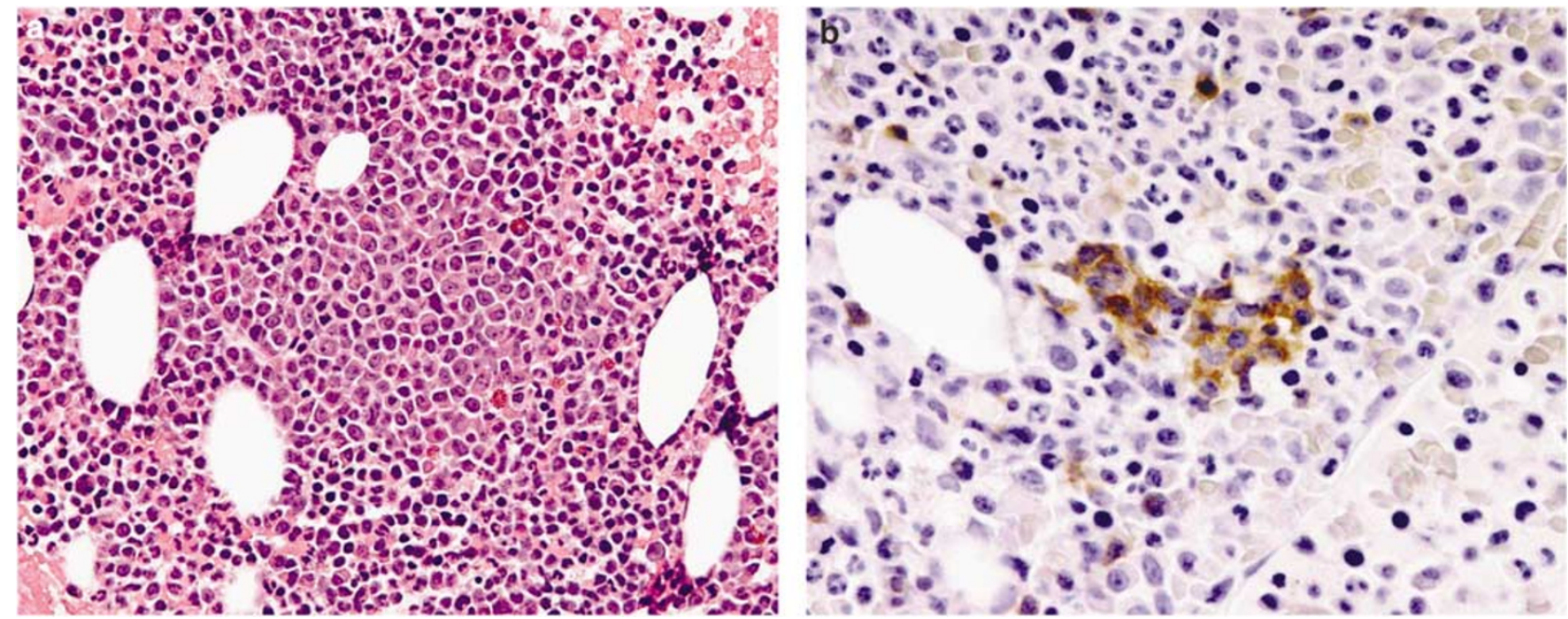

Figure 3 (a) Bone marrow biopsy of a case of CMML. Plasmacytoid monocyte nodules, as the one shown here, are often inapparent in H\&E-stained bone marrow sections. If detected, in the absence of immunohistochemistry, are difficult to distinguish from aggregates of immature myeloid cells. (b) Immunohistochemical staining for CD123 highlights a large nodule of plasmacytoid monocytes and greatly helps in the identification of these cells. 
myelogenous leukemia patients ranged in age from 21 to 60 , their median age was 42 years; four were males and two were females. In the three patients with atypical CML the age ranged from 61 to 87 , their median age was 74 years; all three were males.

Follow-up data were available for most cases. In CMML (non in transformation), three patients died of disease; their survival from diagnosis to death ranged from 5 to 8 months, with a median value of 7 months. The other 20 patients were alive with disease at a median follow-up interval of 11 months (range 2-56). In CMML in acute transformation, all seven patients were alive with disease at a median follow-up interval of 10 months (range 2-23). In the chronic myelogenous leukemia group, two patients died following bone marrow transplant at 3 and 4 months, respectively. Of the remaining four patients, two were in complete remission at 14 and 46 months, from the disease onset, respectively; the remaining two patients had persistent disease at 65 and 70 months from the onset, respectively. The atypical CML patients were alive with disease at a median follow-up of 10 months (range 1-26).

\section{Peripheral Blood Results}

Peripheral blood results are summarized in Table 1. The majority of patients in all group showed mildto-moderate anemia. Anemia was more severe in patients with CMML in acute transformation and atypical CML. The median hemoglobin values were: $10.0 \mathrm{~g} / \mathrm{dl}$ in CMML type-1; $9.7 \mathrm{~g} / \mathrm{dl}$ in CMML type-2; $8.8 \mathrm{~g} / \mathrm{dl}$ in CMML in acute transformation; $10.9 \mathrm{~g} / \mathrm{dl}$ in chronic myelogenous leukemia; and $9.6 \mathrm{~g} / \mathrm{dl}$ in atypical CML. WBC value was variable in CMML cases. The median WBC was: $11.8 \times 10^{9} / \mathrm{l}$ in CMML type- 1 ; $13.5 \times 10^{9} / \mathrm{l}$ in CMML type-2; and $5.5 \times 10^{9} / 1$ in CMML in acute transformation. All cases of chronic myelogenous leukemia and atypical CML showed significant leukocytosis (chronic myelogenous leukemia: median WBC $82.6 \times 10^{9} / 1$; atypical CML: median WBC $48.3 \times 10^{9} / \mathrm{l}$ ). The basophil count was within normal limits or only minimally elevated in both CMML and atypical CML. Basophilia was present in all cases of CML with a median value of $3 \%$. Platelet counts were variable in CMML type-1 and CMML type-2 (median value 185.0 and 180.0, respectively). The lowest platelet counts were observed in patients with CMML in acute transformation (median $28 \times 10^{9} / \mathrm{l}$ ) and atypical CML (median $75 \times 10^{9} / 1$ ). As expected, monocytosis was encountered only in CMML cases. The monocytes showed variable degrees of dysplastic changes, particularly nuclear abnormalities. Dysgranulopoiesis, usually mild, was observed in most cases of CMML but not of chronic myelogenous leukemia. Dysgranulopoiesis was particularly pronounced in the three cases of atypical CML and in the majority of cases of CMML in acute transformation. Rare circulating blasts were found in CMML and chronic myelogenous leukemia patients (median $<1 \%$ ). The blasts were more numerous in cases of CMML type-2 (median 3\%) and CMML in acute transformation (median 2\%). In none of the cases the blasts showed the presence of Auer rods.

\section{Bone Marrow Results}

Bone marrow results are summarized in Table 1. The marrow was hypercellular in the majority of cases of CMML and atypical CML, and hypercellular in all cases of chronic myelogenous leukemia. In all three groups, increased granulocytic proliferation was a constant finding, erythropoiesis was relatively decreased, and megakaryopoiesis was variable. Monocytic proliferation was difficult to detect

Table 1 Peripheral blood and bone marrow aspirate results

\begin{tabular}{|c|c|c|c|c|c|}
\hline Results & $H g b g / d l(m e a n \pm s . d)$. & Plts $\left(10^{9} / 1\right)($ mean \pm s.d.) & $W B C\left(10^{9} / I\right)(m e a n \pm s . d)$. & Mono $\left(10^{9} / 1\right)($ mean \pm s.d.) & Mono (\%) \\
\hline CMML-1 & $10.3(2.1)$ & $175.3(113.6)$ & $21.6(27.9)$ & $5.5(6.4)$ & $28.2(12.4)$ \\
\hline CMML-2 & $9.0(1.1)$ & $227.4(205.2)$ & $36.2(52.8)$ & $4.3(6.3)$ & $14.2(8.1)$ \\
\hline CMML-AT & $8.3(2.0)$ & 49.9 (64.8) & $15.7(21.2)$ & $8.1(14.4)$ & $27.6(21.5)$ \\
\hline CML & $11.5(1.7)$ & $348.1(308.5)$ & $85.2(73.4)$ & $1.1(0.6)$ & $3.5(4.8)$ \\
\hline aCML & $10.1(2.4)$ & $116.6(112.4)$ & $51.9(36.9)$ & $1.6(1.7)$ & $2.6(1.2)$ \\
\hline Results & $P B$ blasts (\%) & BM blasts (\%) & BM mono NBE (\%) & & \\
\hline CMML-1 & $0.4(+0.7)$ & $3.3(+2.2)$ & $15.0(+9.3)$ & & \\
\hline CMML-2 & $3.4(+1.1)$ & $11.4(+0.8)$ & $6.0(+5.7)$ & & \\
\hline CMML-AT & $3.0(+2.0)$ & $35.0(+13.5)$ & $26.0(+19.5)$ & & \\
\hline CML & $1.4(+1.1)$ & $2.1(+1.1)$ & $0.6(+0.9)$ & & \\
\hline aCML & $1.0(+1.0)$ & $3.6(+3.8)$ & $1.1(+1.4)$ & & \\
\hline
\end{tabular}

Hgb g/dl, Plts $\left(10^{9} / \mathrm{l}\right)$, WBC $\left(10^{9} / \mathrm{l}\right)$, Mono $\left(10^{9} / \mathrm{l}\right)$ : the results are expressed as mean value \pm s.d.; the remaining results are in expressed as percentage $(\%)$ values.

Hgb: hemoglobin; Plts: platelets; WBC: white blood-cell count; Mono: monocytes; PB: peripheral blood; BM: bone marrow; BM mono NBE: bone marrow monocytes positive for naphtyl butyrate esterase. CMML: chronic myelomonocytic leukemia; CMML-1: CMML with <5\% blasts in the blood, $<10 \%$ of blasts in the BM. CMML-2: CMML with blasts $5-19 \%$ in the blood or $10-19 \%$ in the bone marrow. CMML-AT: CMML in acute transformation ( $>20 \%$ blasts in the blood and/or bone marrow in a patient with a previous diagnosis of CMML); CML: chronic myelogenous leukemia; aCML: atypical chronic myeloid leukemia. 
Table 2 Bone marrow biopsy: immunohistologic results

\begin{tabular}{|c|c|c|c|c|c|}
\hline Results & $C D 34^{\mathrm{a}}( \pm$ s.d.) & $C D 68 / K P 1^{\text {a }}( \pm s . d)$. & $C D 68 / P G-M 1^{\mathrm{a}}( \pm s . d)$. & $C D 163^{\mathrm{a}}( \pm s . d)$. & Cases pos. for CD123 PM Nodules ${ }^{\mathrm{b}}$ \\
\hline CMML (all) & $4.71(3.7)$ & $30.38(27.7)$ & $3.72(3.5)$ & $2.91(2.1)$ & $6(19 \%)$ \\
\hline CMML-1 & 3.12 (1.9) & $26.9(24.3)$ & $4.3(4.2)$ & $2.53(1.4)$ & $4(21 \%)$ \\
\hline CMML-2 & $11.8(1.3)$ & $56.2(28.7)$ & $2.6(2.1)$ & $4.4(3.5)$ & $2(40 \%)$ \\
\hline CMML-AT & $39.00(20.1)$ & $23.14(29.4)$ & $2.83(0.8)$ & $4.14(4.0)$ & $1(14 \%)$ \\
\hline CML & $3.83(1.9)$ & $90.83(8.7)$ & $2.80(0.8)$ & $2.0(0.6)$ & 0 \\
\hline aCML & $3.6(3.8)$ & $72.3(9.3)$ & $1.0(0.1)$ & $1.0(0.1)$ & 0 \\
\hline
\end{tabular}

${ }^{\mathrm{a}}$ The results are expressed as mean ( \pm s.d.) percentage value of nucleated marrow cells positive with the given marker.

${ }^{\mathrm{b}}$ Cases pos. for CD123 PM nodules: indicates the number of cases positive and in the brackets, the percentage of cases positive for the presence of CD123-positive nodules of plasmacytoid monocytes.

CD68/KP1: CMML, CMML-1, CMML-2 vs CML, $P<0.001$. CD34: CMML vs CMML-AT, $P<0.001$; CMML-1 vs CMML-2, $P<0.05$; CMML-1 vs CMML-AT, $P<0.001$; CML vs CMML-AT, $P<0.01$; aCML vs CMML-AT, $P<0.05$.

morphologically both in the bone marrow aspirate smears and in the histologic sections of biopsy and clot preparation in all cases studied. In cases in which aspirate smears were stained by naphtyl butyrate esterase cytochemistry, a higher proportion of monocytes were identified in CMML (Table 2). The difference between CMML (median naphtyl butyrate esterase positivity, 10\%), chronic myelogenous leukemia (median naphtyl butyrate esterase positivity, $<1 \%$ ), and atypical CML (median naphtyl butyrate esterase positivity, 1\%) was significant (CMML vs chronic myelogenous leukemia: $P<0.001$; CMML vs atypical CML: $P<0.05)$. By careful analysis of the naphtyl butyrate esterase stained smear, a large proportion of the positive cells corresponded to promonocytes. Mature monocytes (the only cell type easily distinguishable in Wright-Giemsa-stained smears) were less constantly present. The blasts counts were increased in CMML type-2 (median 12\%) and CMML in acute transformation (median $35 \%$ ) in comparison with CMML type-1 (median 3\%), chronic myelogenous leukemia (median $2 \%$ ), and atypical CML (median 2\%). Histologically, the blasts were identified mostly by their propensity to form discrete clusters and aggregates. In none of the cases the blasts showed the presence of Auer rods.

Dysgranulopoiesis, which was present in a variable degree in the majority of the CMML patients, was often more evident in the bone marrow than in the peripheral blood. The dysgranulopoietic changes usually consisted of mild abnormalities such as the presence of hypo- or abnormal nuclear lobation and cytoplasmic hypo- or abnormal granularity. Dyserythropoiesis, particularly megaloblastoid changes, were seen in approximately half of the cases of CMML. Dysmegakaryopoiesis, characterized by the presence of dwarf megakaryocytes and/ or megakaryocytes with hypolobated or abnormally lobated nuclei, was found in $75 \%$ of the CMML cases. Similar alterations in the megakaryocytic morphology were also noted in cases of chronic myelogenous leukemia and atypical CML. In chronic myelogenous leukemia, however, a more pronounced degree of megakaryocytic proliferation was often seen. Reticulin staining showed only a modest (1-2+) degree of reticulin fibrosis in most of the cases of CMML and atypical CML. Most cases of chronic myelogenous leukemia showed normal to +1 reticulin fibrosis. The differences between the groups were not significant. None of the marrows included in this series, with the exception of two cases of CMML in acute transformation, showed $>2+$ reticulin fibrosis.

\section{Bone Marrow Biopsy Immunohistology}

The immunohistochemical results are summarized in Table 2.

\section{CD68 (KP1)}

Frequent KP1 reactivity was observed in all groups. The monocytic elements of CMML were often difficult to discern from the granulocytic elements particularly from band forms and metamyelocytes. The highest frequency of positive cells was seen in the cases of chronic myelogenous leukemia. The often hypogranulated neutrophils seen in CMML were only weakly positive or occasionally completely negative with KP1.

\section{CD68R (PG-M1) and CD163}

PG-M1 and CD163 showed comparable results in our series. Both antigens were much more restricted to macrophages and monocytic cells than KP1. The number of reactive cells in CMML cases was small and the differences observed between CMML, chronic myelogenous leukemia, and atypical CML were not statistically significant. When the results obtained with both antibodies were compared to those seen by cytochemistry with naphtyl butyrate esterase, it was apparent that there was a relative lack of sensitivity of the immunohistochemical approach to identifying monocytic cells in paraffin-embedded bone marrow sections in all cases. 


\section{CD34/QBEnd10}

CD34 stained blasts in most cases of CMML type-2 and CMML in acute transformation, and the differences observed between these and chronic myelogenous leukemia, CMML type- 1 and atypical CML CD34-stained marrows were statistically significant. All cases of CML were stable phase disease, a group characterized by low number of CD34-positive marrow cells. ${ }^{19}$ The cases of atypical CML in this study did not have an increased number of marrow blasts, making them indistinguishable from CML and CMML type-1, by CD34 immunostaining. However, owing to small number of cases of atypical CML studied, the CD34 result should not be considered as representative for this disease entity as a whole.

\section{CD123}

Although nodules of plasmacytoid monocytes can be identified morphologically, their identification is much facilitated by immunohistochemistry with CD123. Their presence was restricted to cases of CMML and they were not found in cases of chronic myelogenous leukemia or atypical CML. The plasmacytoid monocytes nodules were composed of variable number of cells and were found scattered throughout the marrow cavity in a nodular/interstitial pattern. The plasmacytoid monocytes were consistently negative with CD163 and variably positively stained with CD68 and CD68R.

\section{CD42b}

CD42b immunostained strongly the megakaryocytes in all the cases. An increased number of dysplastic megakaryocytes (see bone marrow findings) were commonly seen in cases of CMML and atypical CML. The use of CD42b made their recognition easier; however, the differences observed with chronic myelogenous leukemia, a disease also characterized by the presence of small megakaryocytes, were not of a sufficient specificity to allow for a reproducible distinction.

\section{Cytogenetic Results}

Bone marrow karyotype results are summarized in Table 3. Marrow cytogenetics was available in 17 of 20 CMML type-1, in all five cases of CMML type-2, in five of seven cases of CMML in acute transformation, in all cases of chronic myelogenous leukemia, and in two of three cases of atypical CML. Clonal cytogenetic abnormalities were found in five CMML type-1 patients $(29 \%)$. One patient showed loss of chromosome Y only. The other four cytogenetically abnormal patients showed a $\operatorname{del}(12)(\mathrm{p} 11.2 \mathrm{p} 13)$, a $\operatorname{del}(11)(q 21)$, monosomy 7, and trisomy14 abnormalities, respectively. Two of five CMML type-2 had
Table 3 Bone marrow karyotype results

CMML-1

$46, \mathrm{XY}[20]$

46,XX,del(12)(p11.2p13)[20]

$46, \mathrm{XX}[29] /$ Nonclonal[1]

$46, X Y[30]$

$46, \mathrm{XX}[30]$

46,XY[29]/Nonclonal[1]

46,XY,del(11)(q21)[20]

45,XY,-7[3]/Nonclonal[1]/46,XY[2]

$46, X Y[30]$

$46, X Y[31]$

$46, X Y[n a]$

$46, X Y[30]$

$46, X Y[22]$

$46, \mathrm{XX}[20]$

$47, \mathrm{XY},+14[18] / 46 \mathrm{XY}[2]$

$45, \mathrm{X},-\mathrm{Y}[20]$

$46, X Y[n a]$

CMML-2

$46, \mathrm{XX}[20]$

$45, \mathrm{XX},-7[19] / 46, \mathrm{XX}[1]$

$46, \mathrm{XX}[32]$

45,X,-X,?t(3;14)(q13.2;p11.2),-5, del(6)(q15q23),

$-7, \operatorname{hsr}(12)(\mathrm{q} 13), \mathrm{t}(15 ; 21)(\mathrm{p} 11.2 ; \mathrm{p} 13)$,

-18 , add(19)(p13.3),-21,+r,+1 4mar[13]/

$40 \sim 42$, idem,r(7)(p22q36), $-9,-r$,

$-2 \sim 4 \mathrm{mar},+\operatorname{mar} 9[\mathrm{cp} 2] /$ Nonclonal[4]/46,XX[1]

46,XY[19]/Nonclonal[1]

CMML-AT

46,XY[na]

46,XY,i(17)(q10)[15]/nonclonal[1]/46,XY[4]

46,XX,t(11;22)(q24;q11.2)[20]

$46, X Y[20]$

45,XX,t(2;11)(p15;q23),-7,der(11)t(2;11)(p15;q23)

CML

46,XX,t(9;22)(q34;q11.2)[20]

$46, X X, t(9 ; 22)(q 34 ; q 11.2)[20]$

$46, X X, t(9 ; 22)(q 34 ; q 11.2)[20]$

$46, X Y, t(9 ; 22)(q 34 ; q 11.2)$ [na]

$46, X Y, t(9 ; 22)(\mathrm{q} 34 ; \mathrm{q} 11.2)[4] / 46, \mathrm{XY}[16]$

$46, \mathrm{XY}, \mathrm{t}(9 ; 22)(\mathrm{q} 34 ; \mathrm{q} 11.2)[20]$

$a C M L$

46,XY[na]

46,XY,del(6)(q13q23),?add(9)(q22)[11]/

$54, X Y, \operatorname{del}(6)$ (q13q23) X2,?add(9)(q22),+10,+12,+19,+19,+21, $+21[5] /$ Nonclonal[1]/46XY[3]

abnormal marrow karyotypes. One of these had a complex karyotype with, among other abnormalities, monosomy 5 and monosomy 7; the second case showed monosomy 7 only. Of five CMML in acute transformation cases with karyotype results, three were abnormal. These included one case with a I(17)q10, one case with a $t(11 ; 22)(q 24 ; q 11.2)$, and a third case with a $\mathrm{t}(2 ; 11)(\mathrm{p} 15 ; \mathrm{q} 23),-7$, $\operatorname{der}(11) t(2 ; 11)(p 15 ; q 23)$. All the chronic myelogenous leukemia cases showed $t(9 ; 22)$ as a single abnormality; of the two atypical CML analyzed, one showed a complex karyotype which included both a $\operatorname{del}(6)(\mathrm{q} 13 \mathrm{q} 23)$ and a ?add(9)(q22) as well as multiple trisomies (Table 3). 


\section{Discussion}

The true biologic nature of CMML is not well understood. ${ }^{1,20}$ Cases of CMML are cytogenetically heterogeneous, and may include abnormalities such as trisomy 8 , monosomy $7 / 7 \mathrm{q}-$, and abnormalities of $12 \mathrm{p}^{21} \mathrm{~A}$ small proportion of CMML cases are associated with a $\mathrm{t}(5 ; 12)(\mathrm{q} 33 ; \mathrm{p} 13)$ chromosome abnormality involving the PDGFR $\beta$ and TEL genes. ${ }^{22,23}$

Overall, cytogenetical abnormalities are seen $20-40 \%$ of the cases, ${ }^{1}$ a frequency similar to that observed in our series. Cases of atypical CML display a high incidence of complex karyotypes involving both chromosome 5 or 7 , similar to myelodysplastic syndromes and secondary acute myeloid leukemia., ${ }^{1,24}$

In contrast to chronic myelogenous leukemia and a large proportion of the other subtypes of chronic myeloproliferative disorders, the molecular pathogenesis of CMML is still only minimally understood. Mutations of RAS are detected in approximately one-third of CMML cases. ${ }^{25,26}$ Although detection of $R A S$ mutations is not generally useful for diagnosis, abnormalities involving the RAS pathway are thought to be an important mechanism for this and other chronic myeloproliferative disorders, including chronic myelogenous leukemia.

In contrast with the Philadelphia chromosomenegative chronic myeloproliferative disorders, in CMML the JAK2 V617F mutation has not been found to be implicated in the vast majority of cases. In the Philadelphia chromosome-negative chronic myeloproliferative disorders, the JAK2 V617F mutation is thought to contribute to the marrow myeloproliferation by its induction of growth factor independence. ${ }^{27}$

In view of the lack of specific disease marker, the diagnosis of CMML is still largely based on clinicalmorphologic observations. The hybrid nature of CMML with features of both a myelodysplastic syndrome and a myeloproliferative disorder creates both diagnostic difficulties and difficulties in the prediction of clinical behavior. ${ }^{1-4}$ Although other conditions may enter the differential diagnosis of CMML, its primary distinction is, in particular, with chronic myelogenous leukemia and atypical CML. The most important of the conventional diagnostic parameters is the presence of peripheral blood monocytosis, truly the sine qua non of CMML. Except for the monocytosis, these disorders are known to show overlapping clinical and morphologic characteristics. This is particularly true of their bone marrow morphologic characteristics, as also confirmed by this study. Although usually above normal levels, the bone marrow monocyte count has never been shown to be of diagnostic value. It has been suggested that this may be due to the difficulty one has in distinguishing the monocytic population from myelocytes in bone marrows which usually display significant degrees of granulocytic hyperplasia. Several authors have suggested the utility of cytochemical stains for nonspecific esterase (eg naphtyl butyrate esterase) for the purpose of highlighting the increased number of monocytes present in bone marrow aspirates. ${ }^{1,6,28,29}$ However, no adequate information is available concerning the diagnostic value of such approach to confirm a diagnosis of CMML, and its ability to provide a way for separating CMML from other morphologically similar myeloid neoplasms, particularly in cases when peripheral blood results are not available.

Our study has confirmed the previously suspected usefulness of routine nonspecific esterase cytochemical staining to confirm a diagnosis of CMML in bone marrow aspirates. More problematic is the use of bone marrow biopsy for the same purpose. Our study has attempted to circumvent the difficulties in distinguishing myelocytes and monocytic cells in bone marrow biopsy by using immunohistology with markers, which are considered both sensitive and specific for the monocyte/macrophage lineage. However, in spite of this approach, the number of cells identified as monocytic remained low and considerable overlap between CMML and chronic myelogenous leukemia was observed. Although both CD68R (PG-M1) and CD163 were much more restricted to bone marrow macrophages and monocytes than CD68 (KP1), the differences between CMML and chronic myelogenous leukemia were still not significant. Our results in this regard, are thus similar to those observed by others. ${ }^{13-15}$ Paradoxically, CD68 (KP1), an antigen which is localized to lysosomes and neutrophil granules, ${ }^{30,31}$ seems to be more strongly expressed in the myeloid cells of chronic myelogenous leukemia than in CMML. This may be due to the presence of a lesser/ abnormal granularity in the myeloid cells and neutrophils in CMML in comparison with chronic myelogenous leukemia. ${ }^{32,33}$

When results of bone marrow biopsy immunohistochemistry for monocytes were compared to those obtained by staining the bone marrow aspirate by naphtyl butyrate esterase, only a modest degree of correlation was observed with a percentage of monocytes generally higher in the cytochemically stained aspirate smears than in the immunostained biopsies. The most likely explanation is one of a lower sensitivity of CD163 and CD68/PG-M1 antibodies for monocytic cells of CMML, in comparison to cytochemistry. The relative insensitivity of these markers for leukemic monocytic cells as opposed to tissue histiocytes/macrophages has been reported by others. ${ }^{14,15}$ On the contrary, CD123 appeared a potentially useful antibody, given its ability in enhancing the visibility of nodular proliferation of plasmacytoid monocytes, a phenomenon which at least in our small series, was largely restricted to CMML. 
Irrespective of the technique used to assess the degree of monocytic differentiation, the dissociation observed between the peripheral blood monocytosis and the relatively paucicity of those cells in the bone marrow biopsy is nothing new. In 1974, Miesher and Farquet ${ }^{34}$ have described CMML as follows: 'A myelomonocytic variant of leukemia with a predominantly monocytic pattern in the peripheral blood and a predominantly myelocytic pattern in the bone marrow'. This apparent paradox may, perhaps, be explained by a rapid egression from the bone marrow of terminally differentiated monocytes whereas the less mature promonocytes are 'left behind'. This seems to be confirmed by our staining results with naphtyl butyrate esterase, which by highlighting their presence confirms their relative high frequency in cases of CMML. To this regard, it has been recently demonstrated by in vitro studies that in CMML, GM-CSF produced by either autocrine or paracrine mechanisms is the major growth determinant. ${ }^{35}$ Moreover, in some patients with CMML, high serum value of this cytokine has been documented. ${ }^{36}$ It is therefore possible that the mobilizing effects of GM-CSF, which has been shown for monocytes as well as for other myeloid precursors, may play a role by facilitating the egression of monocytes from the marrow and their subsequent localization to the peripheral blood and other tissue.

CD34 immunostaining allowed for a reliable distinction of cases with an increased number of blasts ( $>20 \%$ on the corresponding aspirate), which represent evolution into acute myeloid leukemia. In this regards, our results with CD34 are comparable to those previously obtained by our group as well as by others in myelodysplastic syndromes as well as in cases of chronic myelogenous leukemia. ${ }^{11,19,37,38}$ Therefore CD34 appears useful in the work-up of cases such as CMML, in which the marrow blast count maintains an important prognostic value, as also demonstrated by the inclusion of this parameter in the various staging system employed by the clinical hematologists.

In summary, CMML remains a morphological diagnosis. In the absence of peripheral blood smear demonstrating monocytosis, the bone marrow aspirate smear should be stained with a nonspecific esterase (eg naphtyl butyrate esterase) which, at least in this study, was useful in detecting monocytes and promonocytes, thus separating CMML from the monocytic poor chronic myelogenous leukemia and atypical CML. Although nodules of plasmacytoid monocytes can be identified morphologically and immunostained by CD68, their strong reactivity with CD123 makes their detection much easier. Their presence reinforce a suspected diagnosis of CMML, as there are rarely found in other types of chronic myeloproliferative disorders. CD34 is useful to detect the increased number of blasts seen in aggressive cases of the disease including the cases in transformation to acute myeloid leukemia.

\section{Acknowledgements}

These results were presented in part at the 95th Annual Meeting of the United States and Canadian Academy of Pathology, Atlanta, Georgia, 2006. We also wish to acknowledge Ms Cecilia Dobson for her technical help with immunohistochemistry and Ms Regina Bennett for her secretarial support.

\section{References}

1 Vardiman JW, Imbert M, Pierre R, et al. Chronic myelomonocytic leukemia. In: Jaffe ES, Harris NL, Stein H, Vardiman JW (eds). World Health Organization Classification of Tumors: Pathology and Genetics of Tumours of Haematopoietic and Lymphoid Tissues. Lyon IARC Press: Lyon, France, 2001, pp 49-52.

2 Bennett JM, Catovsky D, Daniel MT, et al. The chronic myeloid leukemias: guidelines for distinguishing chronic granulocytic, atypical chronic myeloid, and chronic myelomonocytic leukemia. Proposals by the French-American-British cooperative leukemia group. Br J Haematol 1994;87:746-754.

3 Fenaux P, Beuscart R, Lai JL, et al. Prognostic factors in adult chronic myelomonocytic leukemia: an analysis of 107 cases. J Clin Oncol 1988;6:1417-1424.

4 Germing U, Gattermann N, Minning H, et al. Problems in the classification of CMML-dysplastic versus proliferative type. Leuk Res 1998;22:871-878.

5 Storniolo AM, Moloney WC, Rosenthal DS, et al. Chronic myelomonocytic leukemia. Leukemia 1990;4: 766-770.

6 Tefferi A, Hoagland HC, Therneau TM, et al. Chronic myelomonocytic leukemia: natural history and prognostic determinants. Mayo Clin Proc 1989;64: 1246-1254.

7 Orazi A, O’Malley DP, Jiang J, et al. Acute panmyelosis with myelofibrosis: an entity distinct from acute megakaryoblastic leukemia. Mod Pathol 2005;18: 603-614.

8 Martiat P, Michaux JL, Rodhain J. Philadelphianegative (Ph-) chronic myeloid leukemia (CML): comparison with $\mathrm{Ph}+\mathrm{CML}$ and chronic myelomonocytic leukemia. Blood 1991;78:205-211.

9 Oscier DG. Atypical chronic myeloid leukemia, a distinct clinical entity related to the myelodysplastic syndrome? Br J Haematol 1996;92:582-586.

10 Manoharan A, Horsley R, Pitney WR. The reticulin content of bone marrow in acute leukaemia in adults. Br J Haematol 1979;43:185-190.

11 Orazi A. CD34 immunoperoxidase staining for the diagnosis of myelodysplastic syndromes and chronic myeloid leukaemia. J Clin Pathol 1995;48:884 (Letter).

12 Kurec AS, Cruz VE, Barrett D, et al. Immunophenotyping of acute leukemias using paraffin-embedded tissue sections. Am J Clin Pathol 1990;93:502-509.

13 Manaloor EJ, Neiman RS, Heilman DK, et al. Immunohistochemistry can be used to subtype acute myeloid leukemia in routinely processed bone marrow biopsy specimens. Comparison with flow cytometry. Am J Clin Pathol 2000;113:814-822.

14 Lau SK, Chu PG, Weiss LM. CD163: a specific marker of macrophages in paraffin-embedded tissue samples. Am J Clin Pathol 2004;122:794-801. 
15 Nguyen TT, Schwartz EJ, West RB, et al. Expression of CD163 (hemoglobin scavenger receptor) in normal tissues, lymphomas, carcinomas, and sarcomas is largely restricted to the monocyte/macrophage lineage. Am J Surg Pathol 2005;29:617-624.

16 Chen YC, Chou JM, Ketterling RP, et al. Histologic and immunohistochemical study of bone marrow monocytic nodules in 21 cases with myelodysplasia. Am J Clin Pathol 2003;120:874-881.

17 Vermi W, Facchetti F, Rosati S, et al. Nodal and extranodal tumor-forming accumulation of plasmacytoid monocytes/interferon-producing cells associated with myeloid disorders. Am J Surg Pathol 2004;28: 585-595.

18 Palmer CG, Blouin JL, Bull MJ, et al. Cytogenetics and molecular analysis of a ring (21) in a patient with partial trisomy 21 and megakaryocytic leukemia. Am J Med Genet 1995;57:527-536.

19 Orazi A, Neiman RS, Cualing H, et al. CD34 immunostaining of bone marrow biopsy specimens is a reliable way to classify the phases of chronic myeloid leukemia. Am J Clin Pathol 1994;101:426-468.

20 Tefferi A. Pathogenetic mechanisms in chronic myeloproliferative disorders: polycythemia vera, essential thrombocythemia, agnogenic myeloid metaplasia, and chronic myelogenous leukemia. Semin Hematol 1999;36: 3-8 (Review).

21 Bacher U, Haferlach T, Kern W, et al. Conventional cytogenetics of myeloproliferative diseases other than CML contribute valid information. Ann Hematol 2005;84:250-257.

22 Golub TR, Barker GF, Lovett M, et al. Fusion of PDGF receptor to a novel ets-like gene, tel, in chronic myelomonocytic leukemia with $\mathrm{t}(5 ; 12)$ chromosomal translocation. Cell 1994;77:307-316.

23 Steer EJ, Cross NC. Myeloproliferative disorders with translocations of chromosome 5q31-35; role of the platelet-derived growth factor receptor Beta. Acta Haematol 2002;107:113-122.

24 van der Plas DC, Grosveld G, Hagemeijer A. Review of clinical, cytogenetic, and molecular aspects of Ph-negative CML. Cancer Genet Cytogenet 1991;52: 143-156 (Review).

25 Plata E, Viniou N, Abazis D, et al. Cytogenetic analysis and RAS mutations in primary myelodysplastic syndromes. Cancer Genet Cytogenet 1999;111:124-129.

26 Paquette RL, Landaw EM, Pierre RV, et al. N-ras mutations are associated with poor prognosis and increased risk of leukemia in myelodysplastic syndrome. Blood 1993;82:590-599.

27 Johan MF, Goodeve AC, Bowen DT, et al. JAK2 V617F Mutation is uncommon in chronic myelomonocytic leukaemia. Br J Haematol 2005;130:968 (Letter).

28 Galton DA. Haematological differences between chronic granulocytic leukaemia, atypical chronic myeloid leukaemia, and chronic myelomonocytic leukaemia. Leuk Lymphoma 1992;7:343-350 (Review).

29 Misialek MJ, Pechet L. A diagnostic dilemma: chronic myelomonocytic leukemia versus atypical chronic myeloid leukemia. A case report and review of the literature. Acta Haematol 1997;98:221-227.

30 Saito N, Pulford KA, Breton-Gorius J, et al. Ultrastructural localization of the CD68 macrophageassociated antigen in human blood neutrophils and monocytes. Am J Pathol 1991;139:1053-1059.

31 Feuk-Lagerstedt E, Samuelsson M, Mosgoeller W, et al. The presence of stomatin in detergent-insoluble domains of neutrophil granule membranes. J Leukoc Biol 2002;72:970-977.

32 Davey FR, Erber WN, Gatter KC, et al. Abnormal neutrophils in acute myeloid leukemia and myelodysplastic syndrome. Hum Pathol 1988;19:454-459.

33 Moretti S, Lanza F, Spisani S, et al. Neutrophils from patients with myelodysplastic syndromes: relationship between impairment of granular contents, complement receptors, functional activities and disease status. Leukemia Lymphoma 1994;13:471-477.

34 Miescher PA, Farquet JJ. Chronic myelomonocytic leukemia in adults. Semin Hematol 1974;11:129-139.

35 Ramshaw HS, Bardy PG, Lee MA, et al. Chronic myelomonocytic leukemia requires granulocytemacrophage colony-stimulating factor for growth in vitro and in vivo. Exp Hematol 2002;30:1124-1131.

36 Geissler K, Ohler L, Fodinger M, et al. Interleukin 10 inhibits growth and granulocyte/macrophage colony-stimulating factor production in chronic myelomonocytic leukemia cells. J Exp Med 1996;184: 1377-1384.

37 Soligo D, Delia D, Oriani A, et al. Identification of CD34+cells in normal and pathological bone marrow biopsies by QBEND10 monoclonal antibody. Leukemia 1991;12:1026-1030.

38 Orazi A, Cattoretti G, Soligo D, et al. Therapy related myelodysplastic syndromes: FAB classification, bone marrow histology, and immunohistology in the prognostic assessment. Leukemia 1993;7:838-847. 